\title{
Age Invaders: Inter-generational Mixed Reality Family Game
}

\author{
E.T. Khoo and A.D. Cheok
}

\begin{abstract}
This paper introduces Age Invaders (AI), a novel interactive inter-generation social-physical game which allows the elderly to play harmoniously together with children in physical space while parents can participate in the game play in real time remotely through the internet.
\end{abstract}

Index Terms-Elderly Entertainment, Mixed Reality, Physical Computing, Social Computing.

\section{INTRODUCTION}

With the increase of technology, there is a huge gap between youth and elderly culture. Present technology does not often allow the facilitation of family entertainment. Most children greatly enjoy playing computer games from childhood and most of them do not like to take part in more elderly-style games such as chess games or fishing. Moreover, most of current computer and video games do not involve physical body movements and social interactions which are proven to be essential for the enjoyment of life [2].

Parents are often busy at work and may have to travel away from the family on business trips. It is important to put in place a social and physical inter-generational family entertainment system that can connect family members in the home and away from the home.

AI is a novel interactive inter-generation social-physical game that allows the elderly to play harmoniously together with children in physical space while parents can participate in the game play in real time through the internet. This game is based on the popular traditional Space Invader [17] arcade game.

The concept of the Age Invaders game is shown in Fig. 1, two children are playing with two grandparents in this interactive physical media space while two parents can join into the game via the internet as virtual players, thus increasing the inter-generational interaction.

The game offers adaptable game parameters to suit the simultaneous gaming of elderly and young. Adjusting game properties automatically compensate for potential elderly disadvantages, for example slower reaction time and slow movement. For example, the rockets of the elderly are faster

Manuscript received on August 20, 2006

E.T. Khoo is with the Mixed Reality Lab, National University of Singapore, 21 Lower Kent Ridge Road, Singapore 119077 (e-mail: khooet @gmail.com).

A.D. Cheok is with the Mixed Reality Lab, National University of Singapore, 21 Lower Kent Ridge Road, Singapore 119077 (e-mail: adriancheok@mixedrealitylab.org).

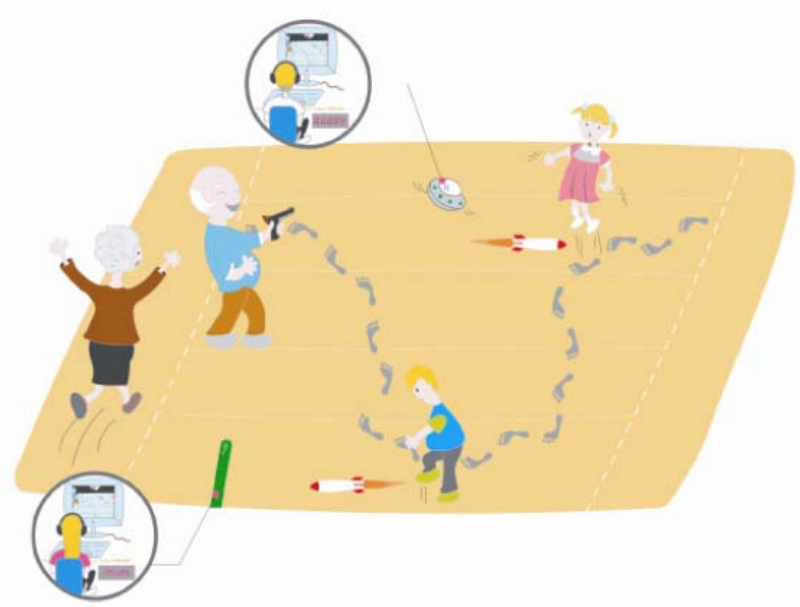

Fig. 1.Age Invaders: An inter-generational, social and physical game. See Color Plate 24

than those of the children's, and vice versa. This property is important to sustain the players' interaction and interest in the game.

Unlike standard computer games, AI requires and encourages physical body movements rather than constraining the user in front of computer for many hours. It also incorporates puzzle solving games which encourage cognitive stimulating activities for the health benefits of the elderly and young.

AI uses a floor display, which is an unconventional interface for mixed reality entertainment. The key advantage of floor display over HMD or other wearable display is that it doesn't require the user to wear something bulky and perceive the world through the device and this is an important feature for elderly to participate in Augmented Reality games. The floor display is very intuitive and provides users with a direct connection to the virtual game world using their whole body as the interface.

Players can move around the game platform as they would for normal activities without having to adapt to new display interface. In real time, as the players move and shoot rockets or bomb, it will appear that it is physically coming out of their bodies, which gives a real time link between the real world and the virtual world. This will immerse the players into the game and introduce high physicality [15], which is important to sustain the players' interest in the game and encourages them to collaborate actively.

\section{BACKGROUND}


There is a global aging population and increasing generation gap between the grandparents or parents and children. In 1950, just 8 per cent of the world population was aged 60 years or over. By 2005 that proportion had risen to 10 percent and it is expected to more than double over the next 45 years, reaching 22 percent in 2050 [21].

The problem of increasing population of the elderly brings along many socio-economic issues. For example, the elderly require increased health cares for both mental and physical problems. Many of these problems derive from social isolation and lack of activity. Findings from scientific research studies show that playing video games can lead to changes in an individual's pleasure, arousal, dominance, and/or affiliative behavior [5, 14, 18]. Furthermore, elderly enjoy computer gaming experience [19]. Therefore it is a highly positive move to use digital new media to increase elderly happiness, through social interaction, physical activity, and entertainment.

Participation in an activity helps elderly to feel better and healthier as they recognize their ability to move and create something which is important to produce a sense of health [13]. According to a recent report, moderate, regular exercise may be just as helpful in combating serious depression in older people as antidepressant medication [8].

On average, compared with someone with the lowest activity level, the risk of Alzheimer's disease [1] was $47 \%$ lower for those whose frequency of activity was highest [20]. Therefore, we believe that cognitive stimulating activities such as Age Invaders could possibly help in part of the prevention process and reverse cognitive impairment in many elderly and also reduces the risk of developing AD.

Often a unique bond connects grandparent and grandchildren [3]. Grandparents can have significant impact on their grandchildren's lives in many different ways. They can act as the family historian, mentor, playmate, nurturer, role model, confidante, advocate, advisor, and surrogate parent. The more grandparents and grandchildren are in contact with each other, the more the relationship grows and the more influence grandparents have [10]. As children nowadays are more inclined to play computer games, it is necessary to create a new platform of interactive games so that the elderly and young can play harmoniously together in a physical space and in long run strengthen the inter-generational bond.

\section{PRIOR RESEARCH}

Very few works have promoted computer entertainment for the elderly, even though it has been shown that elderly enjoy the computer gaming experience [19]. Furthermore, elderly are open to new technology experience: in a study involving seniors at an apartment building, 49\% of the seniors who took part expressed interest in learning about new and more challenging software and 26\% were interested in computer programming [9]. Age Invaders promotes new entertainment computer technology for elderly.
In a study to investigate the possibility that slow-down in performance of the elderly could be reversed through practice on videogames, Pac Man and Donkey Kong which require a measured response selection and have been regarded by elderly as challenging were chosen. The results showed that the weekly high scores on Pac Man nearly tripled over the seven-week period, and Donkey Kong improved almost fivefold [6]. Using entertainment technology, Age Invaders can promote physical and mental benefits for elderly. Previous research in mixed reality gaming has been seen in Human Pacman [4] where the popular Pacman arcade game is adapted into the real world with head mounted displays (HMDs), wearable computers and several sensing mechanisms. While Human Pacman has high social and physical interactions, it has a disadvantage of requiring heavy equipments, sensors and HMDs which is not suitable for elderly people. Age Invaders uses a floor display, which is an unconventional interface for mixed reality entertainment. The key advantage of floor display over HMD or other wearable display is that it doesn't require the user to wear something bulky and perceive the world through the device and these are important features for elderly to participate in Augmented Reality games.

Many inter-generational interactive games developed are in the form of board games. Very little work is found on developing inter-generational computer games. However study shows that greater participation of the elderly and young in computer-related activity benefited both parties [12]. Taken into consideration that difficulty of using keyboard is one of the major problems of elderly using computer, bringing computer games into physical game space could be a smart move.

Thus, by also incorporating inter-generational play, Age Invaders is a mixed reality game aimed to improve the welfare of the elderly using interactive media. We believe that Age Invaders will provide a framework for many inter-generational entertainment technologies in the future such as real time virtual-physical dancing game where the "grandparents" or "parents" will create the sequence of dance steps on the online virtual interface which will appear in real time on the physical game board where the children are dancing on.

\section{BEYOND DIGITAL TRADITION}

Traditional digital games are designed for the young where normally the player sits in front of a computer or game console. Unlike standard computer games, Age Invaders brings the game play to a physical platform, and requires and encourages physical body movements rather than constraining the user in front of computer for many hours. Age Invaders is an interactive social-physical family digital game designed specially for a harmonious game play between the elderly and young. Adjusting game properties automatically compensate for potential elderly disadvantages, for example slower reaction time and slow movement.

Age Invaders is based on the ever popular traditional Space 
The International Journal of Virtual Reality, 2006, 5(2):45-50

Invader [17] arcade game. Space Invaders was designed and programmed by Toshihiro Nishikado for Taito, Japan in 1978 and remains one of the most popular arcade games ever made [7]. Space Invaders has inspired a form of street art, pioneered by the artist Invader [11]. Using ceramic tiles, Invader and other street artists cement together mosaic images of traditional Space Invader aliens, bonus spaceships, and variations on those themes, sometimes including characters from the Pac-Man series of video games. The mosaics, many being small yet others as large as murals, are cemented onto building walls, lamp post bases, and other structures. The form has spread throughout the world and can be found in many cities including Paris, London, New York, Los Angeles, etc. RUBIKCUBISM, featuring mosaics, video installation, and both small and large-scale sculptures exhibited at Sixspace in July 2005, is Invader's first major exhibition in North America [16]. The exhibition showcased the culture of pixelated video game characters. Age Invaders has retained the cultural icon of space invaders and the old analog and pixelated look of the old Space Invaders game. We have decided to use large LED platform as the game's interface to bring out the analog design of the early computer games. The $3 \mathrm{D}$ online virtual interface is a fusion of futuristic 3D space environment with pixelated game characters and large analog LED platform. Age Invaders aims to bridge the young and old culture by bringing the cultural and artistic elements of Space Invaders into modern digital family entertainment. The modern real time mixed reality game concept is easily accepted by the young children. On the other side, the elderly who were once sharing the culture of the early computer games in the 70s and 80s will find the analog and pixelated concept of Age Invaders more relevant and appealing.

Age Invaders also introduces performance play element. It is a new kind of entertainment where the game player also doubles as a performer. The players have to follow a predefined pattern which will light up on the electronic game floor in a given time, or else the health level of the player will drop quickly. This is an electronic pattern which they will see on the floor. Again we balance the game play, by making the pattern to be followed automatically more difficult for younger players, and easier for older players. This dance like movements introduced by the floor patterns has a performance element which would attract the audience's attention and amusement. Instead of passively watching others playing computer games, in our system the audience could enjoy an immersive experience by watching the performance offered by this interactive media.

\section{SYSTEM DESCRIPTIONS}

The concept of the Age Invaders game is shown in Fig. 2, two children are playing with two grandparents in this interactive physical media space while two parents can join into the game via the internet as virtual players, thus increasing the inter-generational interaction.

Age Invaders system architecture is show in Fig. 3. The system was designed to track players' positions in real time us

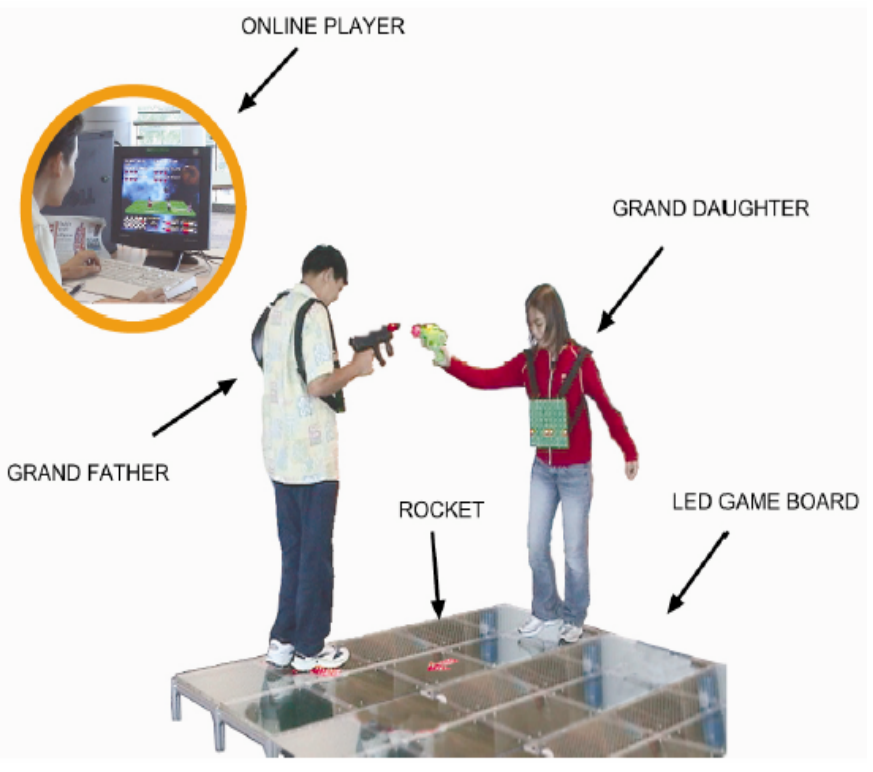

Fig. 2.Age Invaders: An inter-generational, social and physical game. See Color Plate 25

-ing RFID reader embedded in the players' shoes. A large electronic game board made from high resolution LED blocks is where the game will be played on. In real time as players move and shoot using the Bluetooth toy gun, rockets will be shown moving out from the blocks on which they are standing. Players can trigger rockets and bombs to the other players and collect extras to gain or lose score points. Upon hit by rocket, the wireless LED will display explosion pattern (Fig. 4). Player can avoid getting hit by jumping over the approaching rocket to another sub panel (Fig. 5). Gaining the most points in a set amount of time wins the round.

Bonus items can also be placed and explosions triggered by the virtual players logged in the system through Internet. The virtual players could interact strongly by choosing which player to help and picking up bonus items for this player (Fig. 6). They can also adjust the game parameters (e.g. speed of the rockets) by dragging the slide bar. More importantly, the virtual players can see a real time representation of the physical board space, thus increasing the link between the real and virtual world as shown in Fig. 7.

\section{CONCLUSION}

In this project we have developed a system for mixed reality family entertainment. The system features a large floor display platform which is synchronized with the $3 \mathrm{D}$ virtual online interface in real time, real time position tracking of players, real time remote internet gaming, and automatic adjustment of game parameters based on customizable game functions which define the relationships among game difficulty, response speed, and the age. Players have to key in their name and age into the server and the game parameters will be automatically balanced by our system to compensate for the potential elderly 
The International Journal of Virtual Reality, 2006, 5(2):45-50

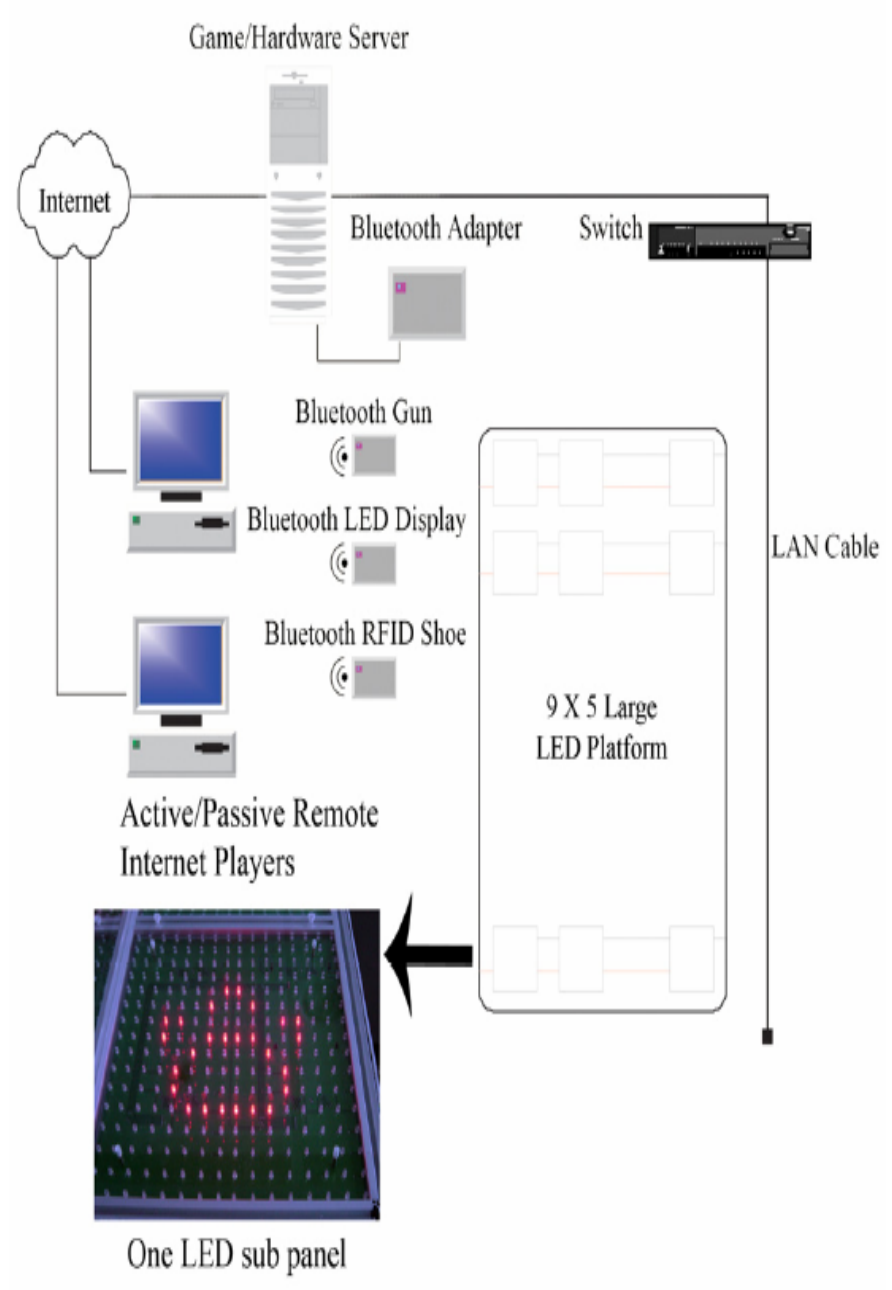

Fig. 3. System architecture of Age Invaders.

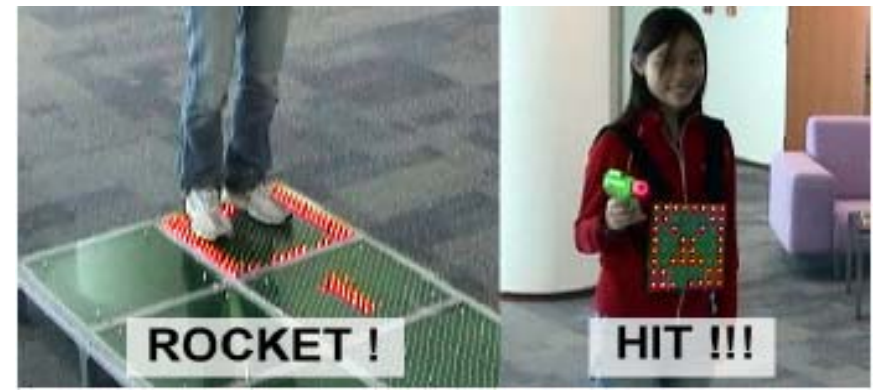

Fig. 4.Grandchild is hit by the rocket and explosions displayed on LED jacket.

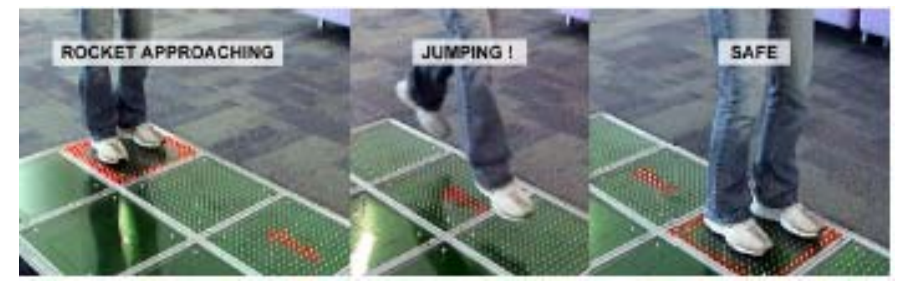

Fig. 5. Dodging the rocket by hopping over it.
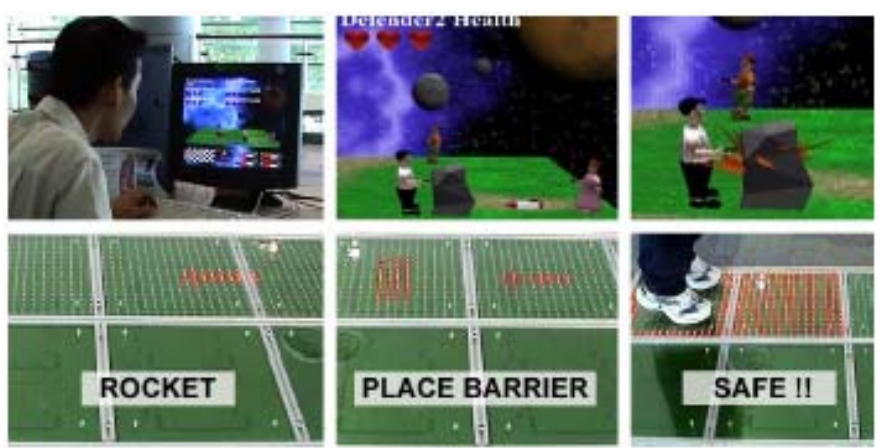

Fig. 6.Grandparent in physical space is saved by barrier dropped in virtual space.

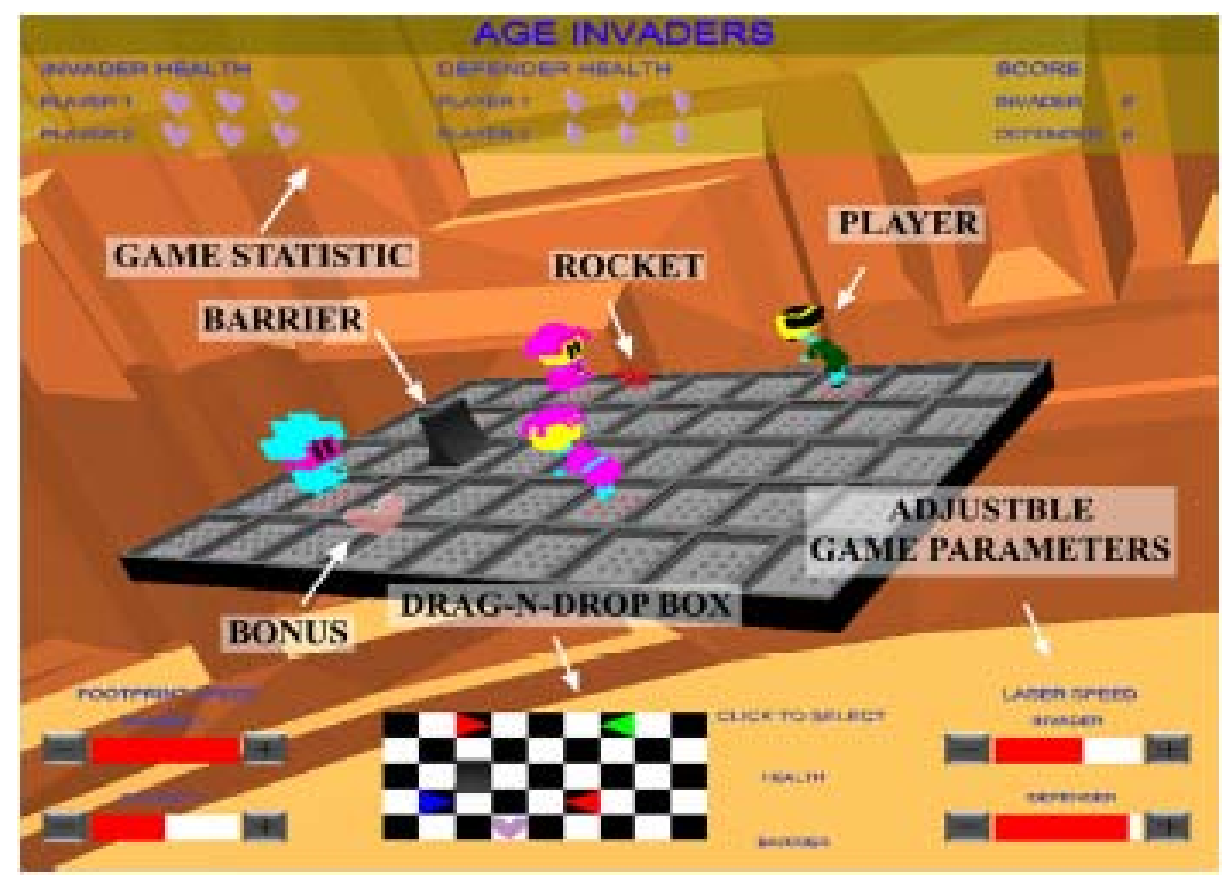

Fig. 7. 3D virtual player interface linking virtual and real physical game world. 
The International Journal of Virtual Reality, 2006, 5(2):45-50

disadvantages. These game parameters are dynamic and are ad -justable by the remote virtual internet players during the game play, in real time. The system's inter-devices communication is completely wireless with wireless toy guns, wireless body display and wireless RFID reader shoes for real time position tracking of players.

Age Invaders uses a floor display, which is an unconventional interface for mixed reality entertainment. The key advantage of floor display over HMD or other wearable display is that it doesn't require the user to wear something bulky and perceive the world through the device and this is an important feature for elderly to participate in Augmented Reality games. The floor display is very intuitive and provides users with a direct connection to the virtual game world using their whole body as the interface. Large LED display platform is chosen over projection display to retain the analog feel of the Space Invaders game. At the same time the LED floor display is not affected by shadow of players moving on the platform.

The main goals of Age Invaders for the elderly generally focus on four major areas: social, physical, cognitive and psychological. The social aspect emphasizes family and inter-generational social interaction, sharing and support. The physical aspect attends to the aging individual's need for physical exercise and expression. The cognitive aspect stimulates the mental functioning and improves the elderly adult's mental stimulation. The psychological aspect refers to promoting personal integration, to the expression of emotions, and feelings of self-worth and wellbeing in a family interaction context.

\section{REFERENCES}

[1] Alzheimer. Information about alzheimer's disease. http:// www. alzheimers. org/

[2] J. Bowlby, Attachment and loss, volume i: Attachment. Basic Book, New York, 1983

[3] L. Carson, The essential grandparent. Deerfield Beach: Health Communications, Inc., 1996

[4] A. D. Cheok, S. W. Fong, K. H. Goh, X. Yang, W. Liu, F. Farbiz and Y. Li. Human pacman: A mobile wideare entertainment system based on physical, social and ubiquitous computing, Personal Ubiquitous Computing ,vol.8, no.2, pp.71-81, 2004

[5] H. Christian, An activity analysis of electronic game simulators, Therapeuticrecreation Journal, vol.12, pp.21-25, 1978

[6] J. E. Clark, A. K. Lanphear and C. C. Riddick. The effects of video game playing on the response selection of elderly adults, Journal of Gerontology vol.42, no.1, pp.82-85, 1987

[7] CLASSICGAMING. History: Space invaders. http: // www. classicgam -ing.cc/ classics /spaceinvaders/history.php

[8] Exercise Works in Treating Elderly Depression. http: // www. healthy -place.com/communities/depression/elderly_3.asp

[9] E. K. Hollander and H. R. Plummer. An innovative therapy and enrichment program for senior adults utilizing the personal computer, Activities, Adaptations and Aging, vol.8, no.1, pp.59-68, 1986

[10] Importance of Grandparents to Their Grandchildren. http://www. foreverfamilies.net/xml/articles/grandparents_importance.aspx/

[11] INVADER. http://www.space-invaders.com.

[12] K. aycox, B. Hicks, Elders. Students and Computers, University of Illinois, 1976
[13] M. Matsuo, J. Nagasawa, A. Yoshino, K. Hiramatsu, K. Kurashiki. Effects of Activity Participation of the Elderly on Quality of Life, Yonago Acta medica, 2003

[14] F. McGuire. Improving the quality of life for residents of long term care facilities through videogames, Activities, Adaptation \& Aging, vol.6, pp.1-7, 1984

[15] S. Price, Y. Rogers. Let's get physical: the learning benefits of interacting in digitally augmented physical spaces, In Computers \& Education, vol. 43, pp.1-2 \&pp.137-151, 2004

[16] SIXSPACE. Invader: Rubikcubism - a logical exhibition, http://six -space .com/gallery/invader2005

[17] Space Invader. http://www.spaceinvaders.de

[18] S. Weisman. Computer games and the frail elderly, Gerontologist, vol. 23, pp.361-363, 1983

[19] G. R. Whitcomb. Computer games for the elderly, ACM SIGCAS Computers and Society archive, vol.20, no.3, pp.112-115, 1990

[20] R. S. Wilson, C. F. M. Deleon, L. L. Barnes, J. S.Schneider, J. L.Bienias, D.A.Evans and D.A.Bennett. Participation in cognitively stimulating activities and risk of incident alzheimer disease, JAMA 287, pp. 742 748, 2002

[21] World Population Prospects: The 2004 Revision. United Nations Department of Economic and Social Affairs’ Population Division, 2005

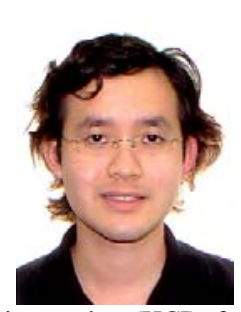

E.T. Khoo is a research scholar pursuing his postgraduate degree at Mixed Reality Lab in National University of Singapore. He joined the research center in July 2005. He was previously a recipient of the ASEAN scholarship. He was studying at the National Junior College and at the National University of Singapore for his Electrical Engineering Bachelor's degree (Hons).

His main research topics are human-computer interaction (HCI), family entertainment, interactive language with music and interactive music with visual art.

He is exclusive composer of Rock Publishing (滚石), a major Chinese songs recording label from Taiwan. He has authored and co-authored several conference papers and journal papers. He has shown his works, Age Invaders, in Montreal, CHI 2006, the premier HCI conference in the world and in Tokyo, Interactive Tokyo 2006.

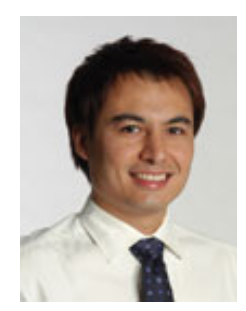

A.D. Cheok is Director of the Mixed Reality Lab, National University of Singapore. He is Associate Professor in the Department of Electrical and Computer Engineering.

He has previously worked in real-time systems, soft computing, and embedded computing in Mitsubishi Electric Research Labs (Osaka, Japan) and NUS. He has been working on research covering mixed reality, human-computer interaction, wearable computers and smart spaces, fuzzy systems, embedded systems, power electronics, and multi-modal recognition. He has successfully obtained funding for four externally funded projects in the area of wearable computers and mixed reality from the Defense Science Technology Agency Singapore. The research output has included numerous high quality academic journal papers, research prototype deliverables to DSTA, numerous demonstrations including to the President and Deputy Prime Minister of Singapore, CNN / CNBC television worldwide broadcasts on his research, and international invited new media exhibits such as Ars Electronica.

He is currently an Associate Professor at the National University of Singapore where he leads a team of over 20 researchers and students. He has been a keynote and invited speaker at numerous international and local conferences and events. He is invited to exhibit for two years in the Ars Electronica Museum of the Future, launching in the Ars Electronica Festival 2003. He was IEEE Singapore Section Chairman 2003, and is presently ACM SIGCHI Chapter President. He was awarded the Hitachi Fellowship 2003, the 
The International Journal of Virtual Reality, 2006, 5(2):45-50

A-STAR Young Scientist of the Year Award 2003, and the SCS Singapore Young Professional of the Year Award 2004. In 2004 he was invited to be the Singapore representative of the United Nations body IFIP SG 16 on Entertainment Computing and the founding and present Chairman of the Singapore Computer Society Special Interest Group on Entertainment Computing. Also in 2004, he was awarded an Associate of the Arts award by the Minister for Information, Communications and the Arts, Singapore. 